The remaining chapters are in more specialised fields, such as cell-surface receptors and neurotransmitters (Michell), macromolecular aspects of nervous communication (Lagnado and Beale), complement (Reid), photosynthesis (Gregory) and vision (Voaden). Several of these authors offer aperitifs of relevant background biology that whet the appetite for their main course. Laskey writes most lucidly on the biochemical basis of cell specialisations in early embryonic development. He emphasises DNA replication, transcription and protein synthesis with an analytical approach that is free from unprofitable speculation. Intermediary metabolism of animals is not given a chapter and plants get only one, on photosynthesis.

I am sure that this book will be read, studied and enjoyed with much profit by advanced students and teachers of biochemistry, even though some chapters are already somewhat obsolete, so raising the question whether quicker ways of production cannot be found for this type of material. Although there is no use of colour, the presentation is generally good; the type is clear, and there are many good line diagrams and a full subject index. Photographic material is well-chosen, though the quality of reproduction might have been better. The cost per page is not unreasonable by comparison with other publications of similar purpose, such as Essays in Biochemistry.

Kenneth Burton is Professor of Biochemistry at the University of Newcastle upon Tyne, UK.

\section{Ocean sound speed fluctuations}

\section{John A. DeSanto}

Sound Transmission through a Fluctuating Ocean. Edited by S. M. Flatte. Pp. 299. (Cambridge University Press: Cambridge, 1979.) $£ 17.50$.

THE book is a very well-edited collection of results arising out of the Jason group of the Advanced Research Projects Agency. It presents their theoretical treatment of the effects of a particular model of ocean sound speed fluctuations (due solely to internal waves) on acoustic propagation, and compares the results with those of three experiments, one in the Western Atlantic (Eluthera-Bermuda), one in the Eastern Atlantic (Azores), and one in the North-eastern Pacific (Cobb Seamount).

Both deterministic and statistical properties of the ocean differ from those of other media. Deterministically, the ocean is refractive in depth (unlike atmospheric optics). Statistically, ocean fluctuations differ from those of homogeneous isotropic turbulence, for example, by being anisotropic, inhomogeneous and by having a different spectral function.

The effects on an acoustic signal propagating through the random ocean region depend on the size (diffractive effect) and strength (simply, the rms phase variation) of the random sound speed inhomogeneities. If the region is a weak scatterer ('unsaturated') neither signal amplitude nor phase fluctuate rapidly. For a stronger scatterer ('partially saturated') the phase begins to fluctuate rapidly, and for a very strong scatterer ('saturated') both phase and amplitude fluctuate rapidly. Also, the longer the propagation range and/or the higher the frequency the more the saturation.

The book contains several parts. Part I is on the ocean environment (ocean structure, planetary waves and eddies, and linear internal waves), and is an excellent introduction to the physics of the ocean and the internal wave fluctuation model used throughout. The fluctuations are thus modelled as arising solely from that spectral window of ocean variability bounded below by the Coriolis (inertial) frequency and above by the Vaisală (buoyancy) frequency.

In Parts II, III and IV, the theoretical methods of sound transmission are developed and interrelated. Path-integral methods (useful in the saturated region), the Rytov approximation (unsaturated region), and the parabolic approximation are all developed, with particular attention paid to ray-theory methods. Of particular use is the discussion of the statistics of acoustic signals in regimes of different saturation.

Do internal waves account for acoustic fluctuations? Part $V$ compares the theoretical predictions to the three experiments. With this extensive and careful development one rather tends to expect a smashing success. It isn't so. The results are mixed. For the (saturated)
Eluthera-Bermuda experiment the rms phase rate and Cartesian signal statistics are predicted well, phase and intensity spectra poorly. For the Cobb Seamount experiment (unsaturated) the internal wave model doesn't account for the measured intensity fluctuations. For the Azores experiment (partial saturation) the agreement is generally good.

My opinion is that the mixed results should not detract from this well written book. It contains a great deal of information, a consistent focused methodology, and the starting point for much additional work. It is suited to an advanced graduate course; and its balance of theory, computations and experimental comparison can serve as a model for extension to the effects of other ocean spectral regimes on acoustic propagation.

John A. DeSanto is a research physicist at the Naval Research Laboratory, Washington, DC.

\section{Geothermal energy}

\section{E. R. Oxburgh}

Geothermal Energy. By H. Christopher H. Armstead. Pp.357. (E. and F. N. Spon: London, 1978.) $£ 10.50$.

OF THE 'alternative energy' resources attracting attention in the early seventies at the time of the rapid rise in world oil prices, geothermal energy is probably the only one for which the expectations are better today then they were five years ago. On the other hand, it must be admitted that at that time expectations were rather low, certainly in Britain.

The exploitation of geothermal energy involves extracting water from sufficiently deep in the ground that it arrives at the surface hot enough for useful heat to be obtained from it. In geothermal prospecting the aim is to identify places where subsurface temperatures are abnormally high and where, at the same time, the rocks are sufficiently permeable to permit the circulation of water through them. Although in principle any desired temperature up to about $250^{\circ} \mathrm{C}$ could be reached by deep drilling, in many places this value would be reached only at depths of 10-12 kilometres, close to the depth limits of present drilling technology. Drilling costs are so high, however, that at present the economic viability of a geothermal prospect depends on whether water at useful temperatures can be obtained from depths of less than about 4 kilometres. Of the geothermal power in use today (providing about $0.25 \%$ of the world's electrical energy) the greater part is derived from much shallower depths than this; in volcanic areas it is often possible to work with holes of the order of a kilometre deep. 